\title{
Anew Synthetic Polymers Based On Polyaniline For dual Functional Applications: Photoelectrochemical Water Splitting And Antibacterial Activities
}

Reem M Abdelfattah', Mohamed Shaban ${ }^{2,3}$, Fatma Mohamed ${ }^{1,2 *}$, Ahmed A.M. El-Reedy ${ }^{4}$ and Hanafy M Abd El-Salam ${ }^{1}$

${ }^{1}$ Department of Chemistry, Faculty of Science, Beni-Suef University, Beni-Suef 62514, Egypt.

${ }^{2}$ Nanophotonics and Applications Lab, Faculty of Science, Beni-Suef University, Beni-Suef 62514, Egypt.

${ }^{3}$ Department of Physics, Faculty of Science, Islamic University in Almadinah Almonawara, Almadinah, 42351, Saudi Arabia

${ }^{4}$ Basic Science Department, Faculty of Oral and Dental Medicine, Nahda University, Beni Suef, Egypt. 


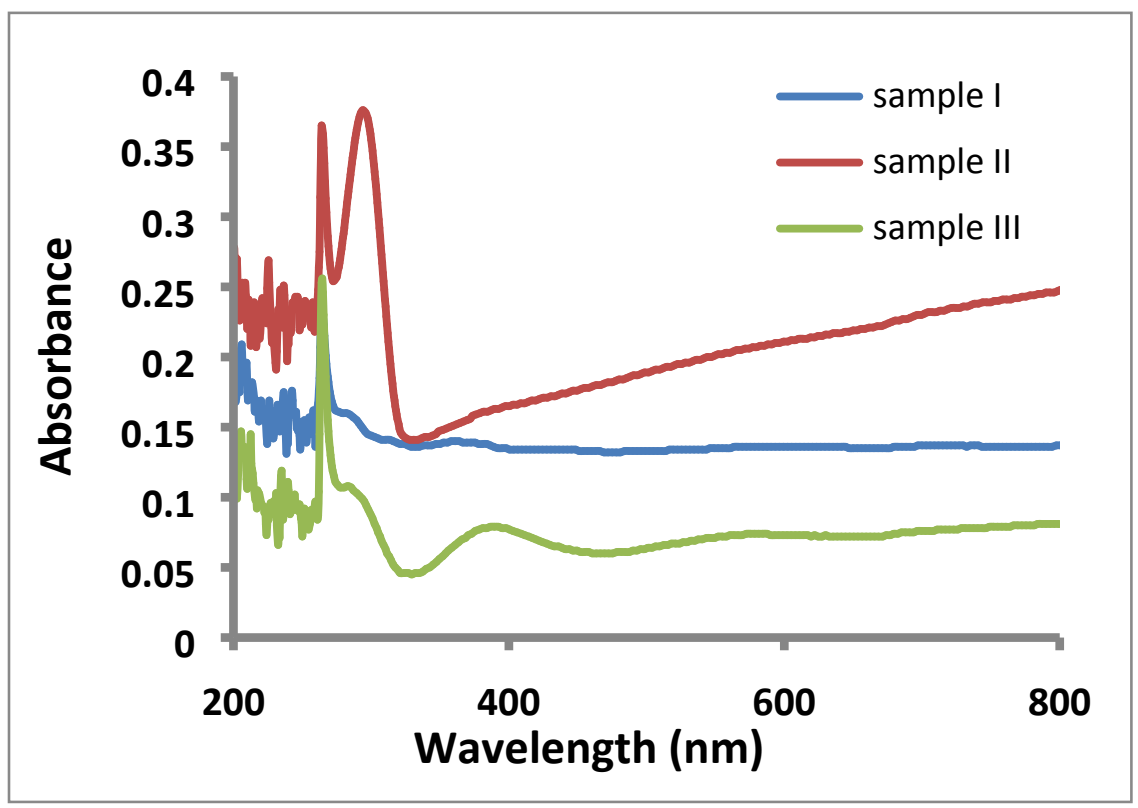

Figure S1. UV spectra of three polymeric samples I, II, III.

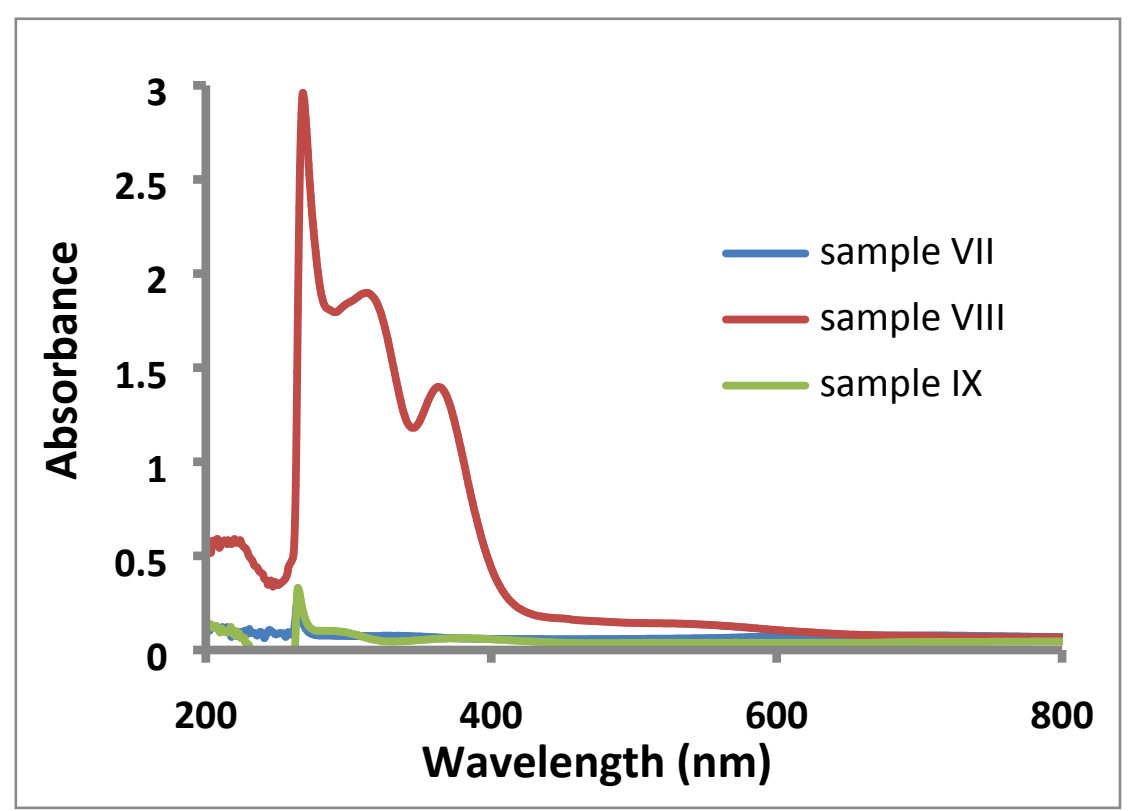

Figure S2. UV spectra of three polymeric samples VII, VIII, IX. 


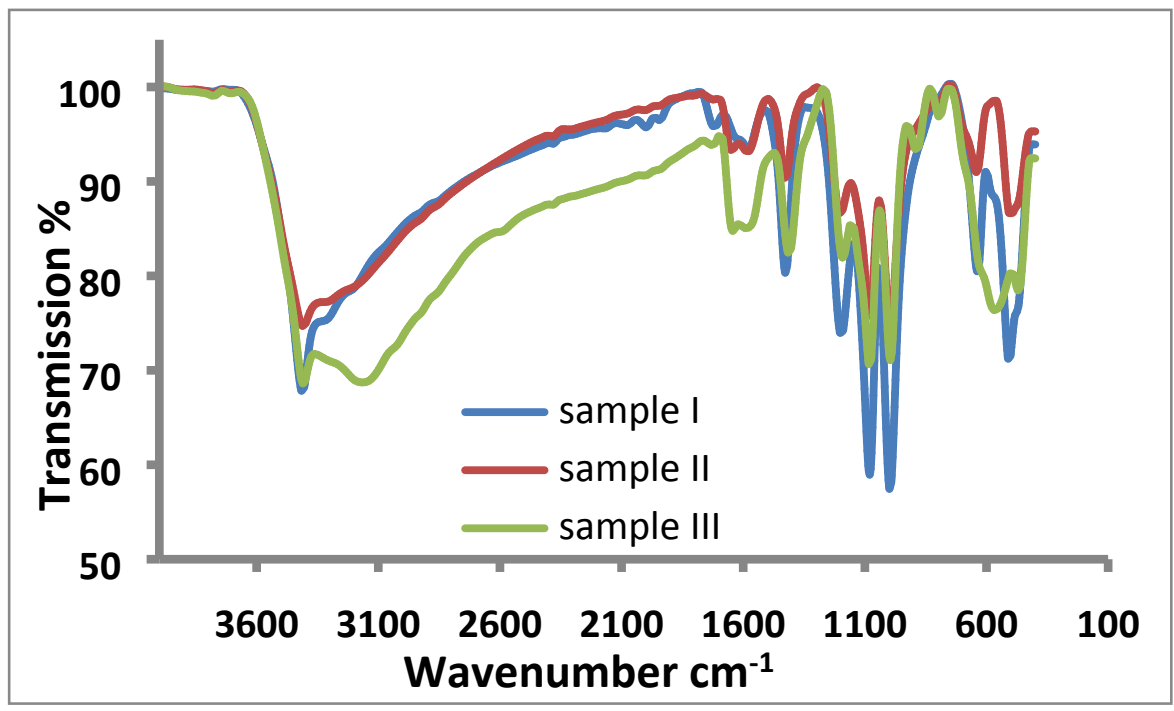

Figure S3. IR spectra of three polymeric samples I, II, III.

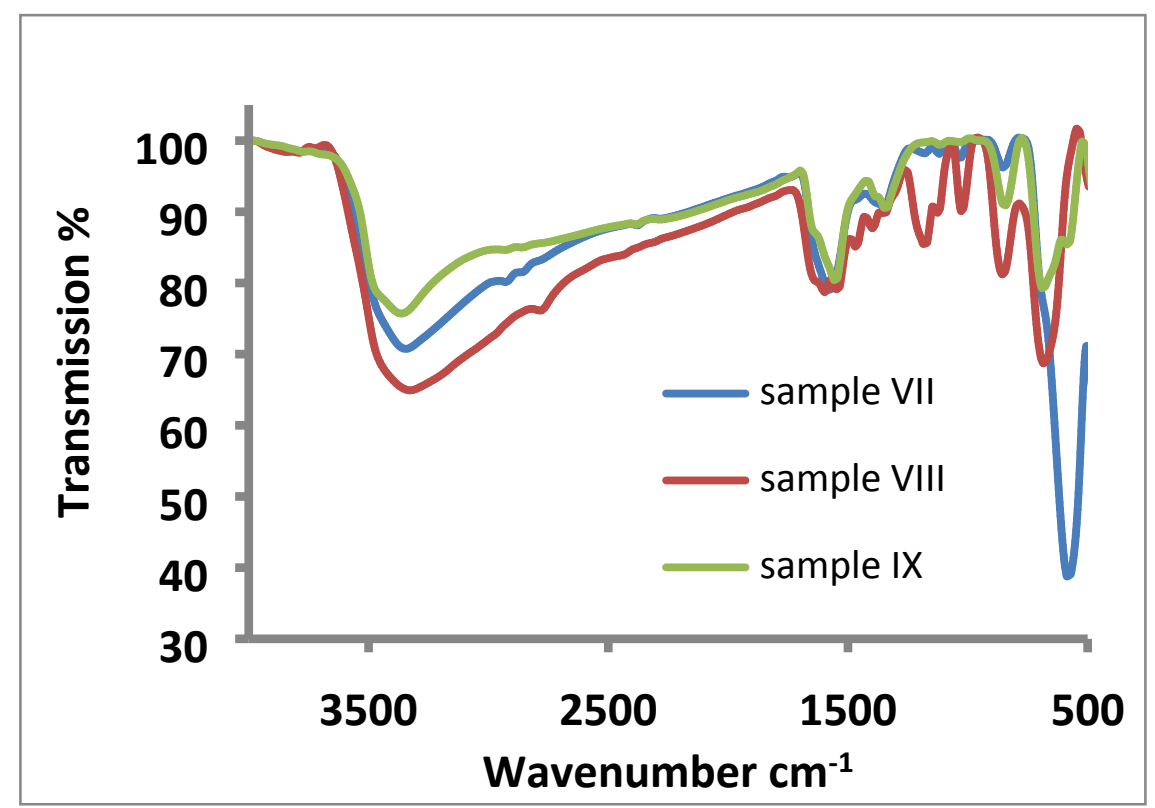


Figure S4. IR spectra of three polymeric samples VII, VIII, IX.
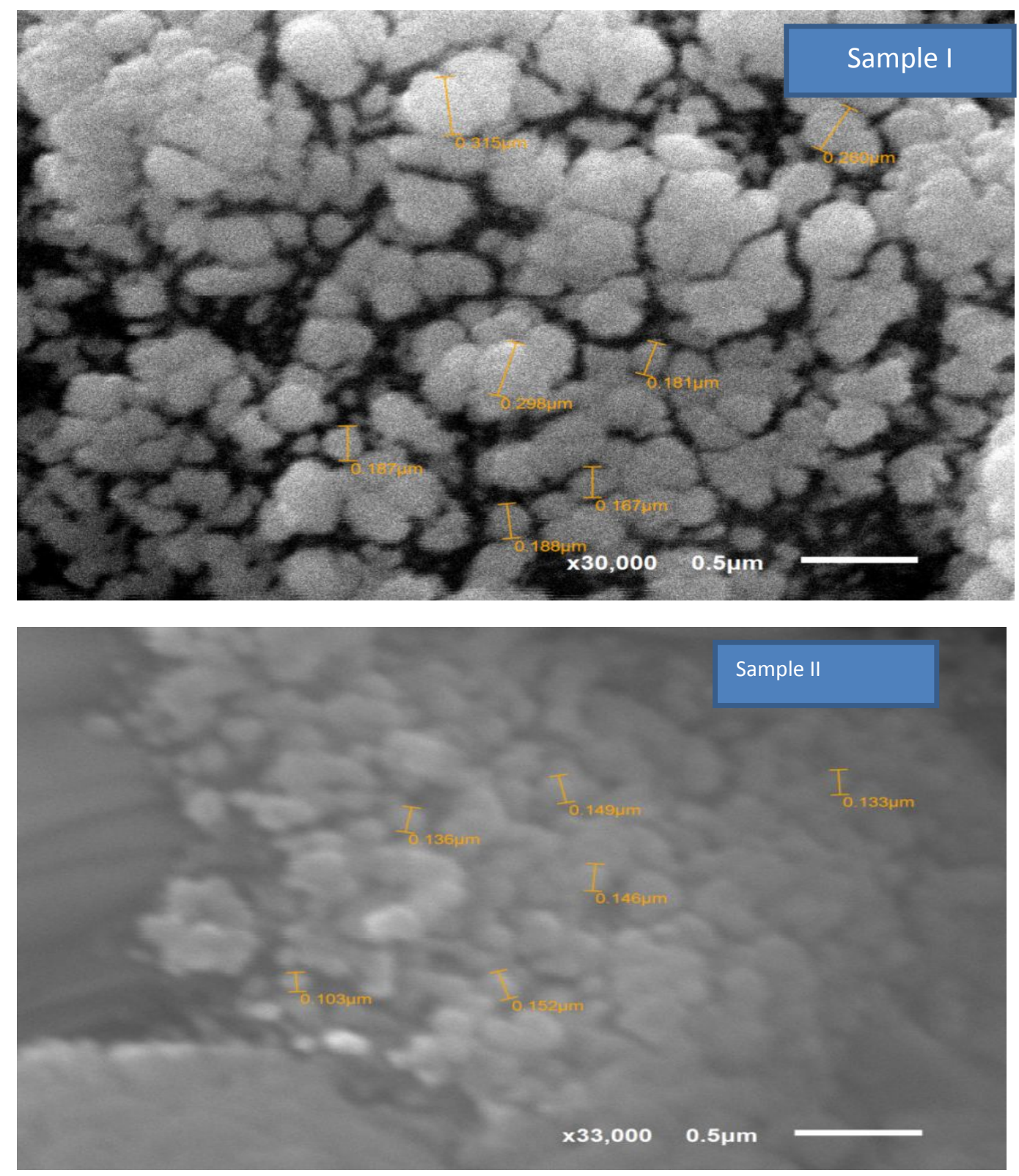


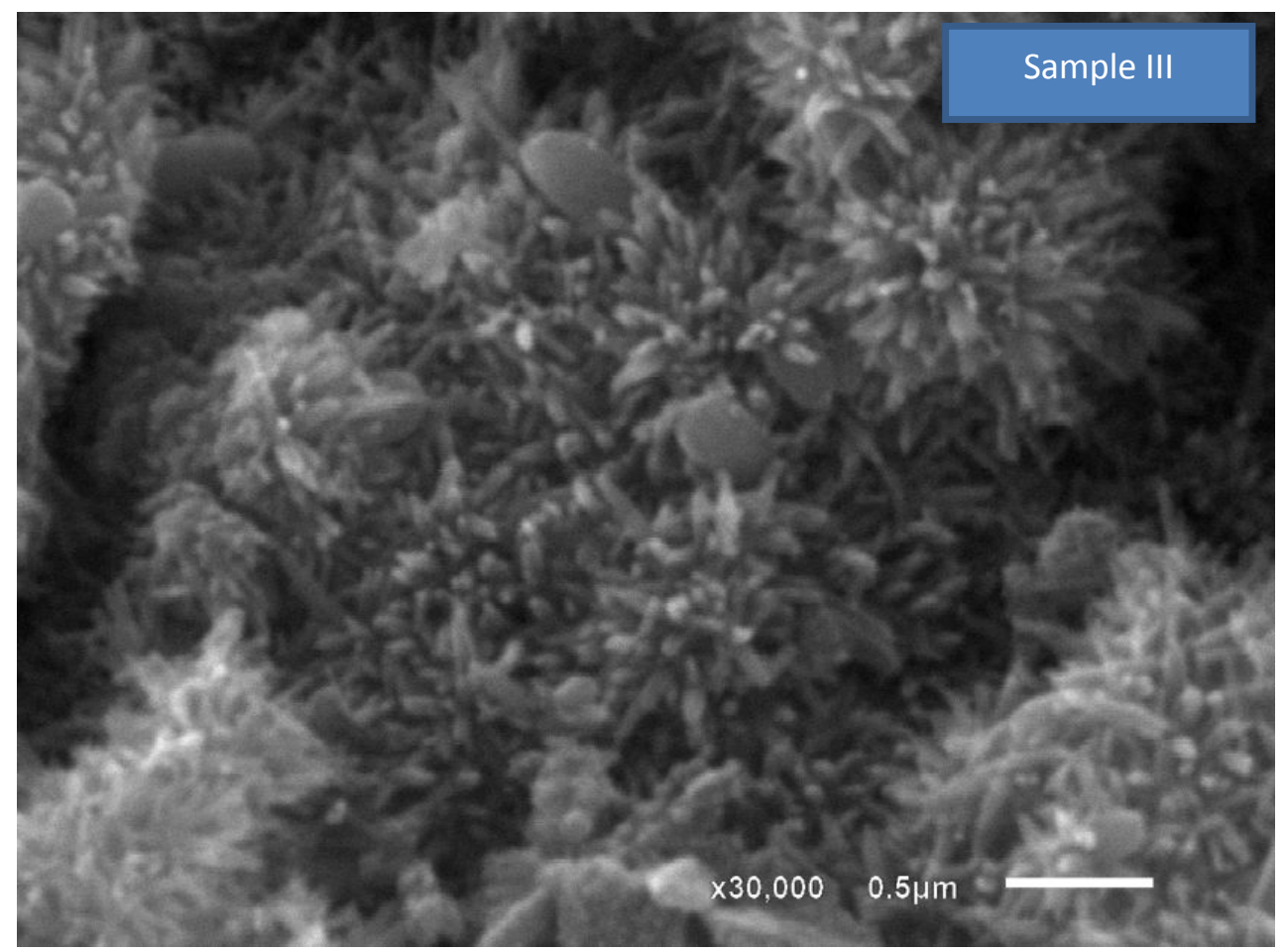

Figure S5. SEM images of three polymeric samples I, II, III. 

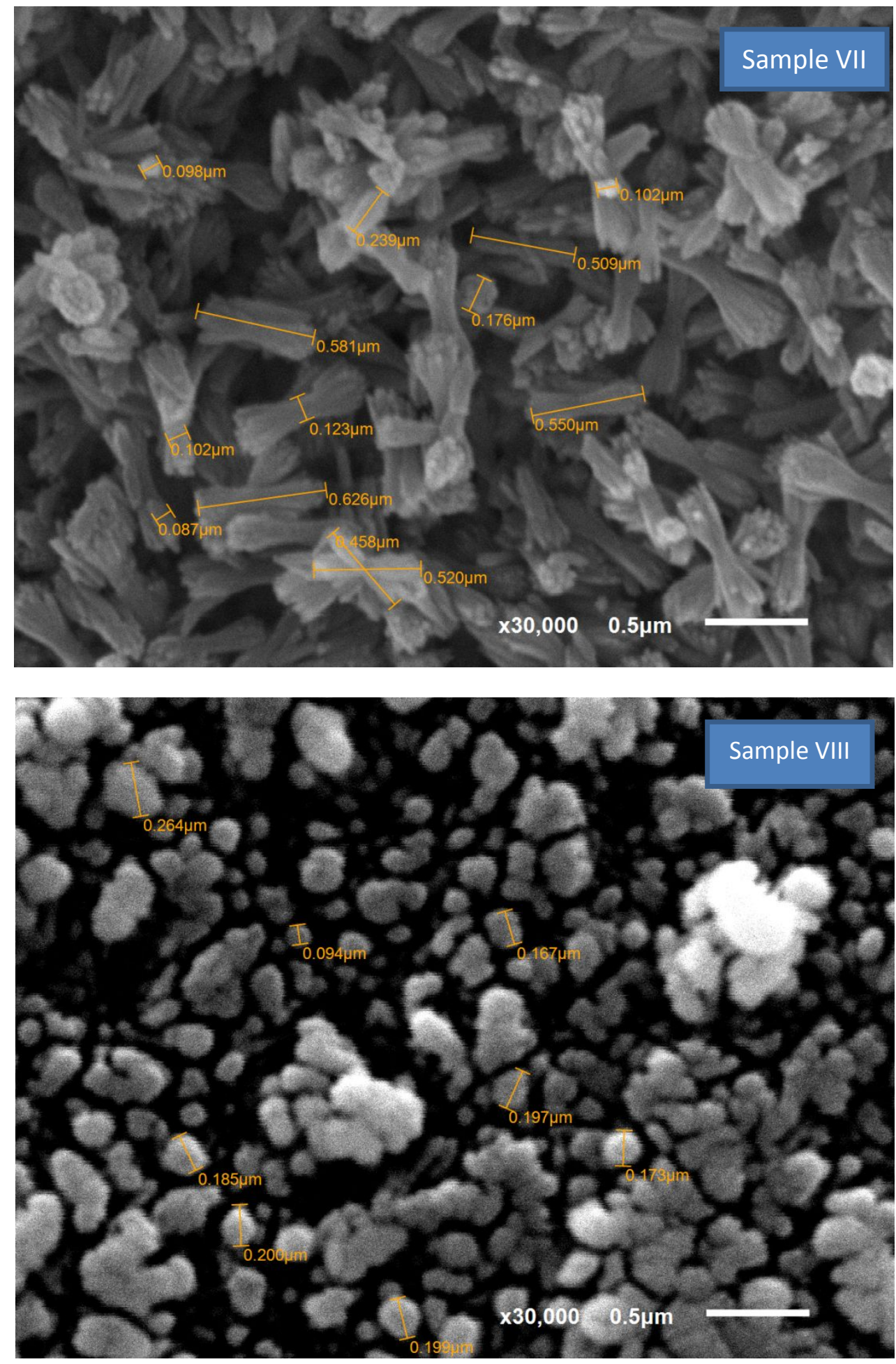


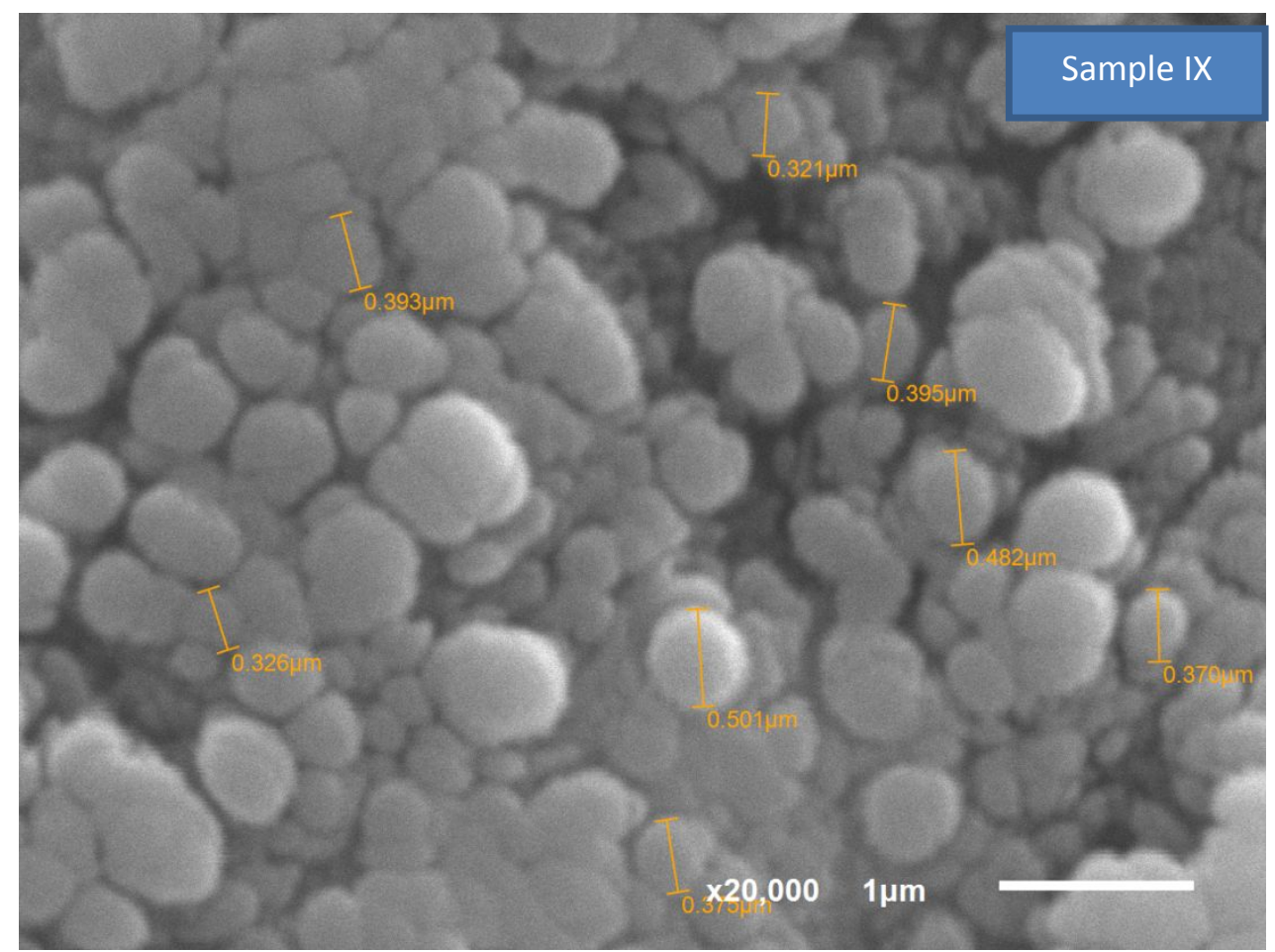

Figure S6. SEM images of three polymeric samples VII, VIII, IX.

Table S1. Antimicrobial activity of the tested samples measured by (mm)

\begin{tabular}{|l|l|l|l|l|l|l|}
\hline $\begin{array}{l}\text { Test organism// } \\
\text { Compound }\end{array}$ & $\begin{array}{l}\text { Bacillus } \\
\text { subtilis }\end{array}$ & $\begin{array}{l}\text { Staph. } \\
\text { aureus }\end{array}$ & $\begin{array}{l}\text { Escherichia } \\
\text { coli }\end{array}$ & $\begin{array}{l}\text { Pseud. } \\
\text { aeruginosa }\end{array}$ & $\begin{array}{l}\text { Candida } \\
\text { albicans }\end{array}$ & $\begin{array}{l}\text { Aspergillus } \\
\text { niger }\end{array}$ \\
\hline SA oligomers & 13 & 13 & $-\mathrm{ve}$ & $-\mathrm{ve}$ & $-\mathrm{ve}$ & $-\mathrm{ve}$ \\
\hline $\begin{array}{l}\text { ANI+SA } \\
\text { Copolymer }\end{array}$ & 22 & 22 & 19 & 20 & 21 & 23 \\
\hline PANI+PS blend & 21 & 22 & 20 & 21 & 20 & 22 \\
\hline Reference & 29 & 28 & 28 & 30 & 27 & 30 \\
\hline
\end{tabular}

Table S2. Minimum inhibitory concentration (MIC) of the tested samples measured by (ppm) 


\begin{tabular}{|l|l|l|l|l|l|}
\hline $\begin{array}{l}\text { Test organism/ } \\
\text { Compound }\end{array}$ & $\begin{array}{l}\text { Bacillus } \\
\text { subtilis }\end{array}$ & $\begin{array}{l}\text { Staph. } \\
\text { aureus }\end{array}$ & $\begin{array}{l}\text { Escherichia } \\
\text { coli }\end{array}$ & $\begin{array}{l}\text { Pseud. } \\
\text { aeruginosa }\end{array}$ & $\begin{array}{l}\text { Candida } \\
\text { albicans }\end{array}$ \\
\hline $\begin{array}{l}\text { ANI+SA } \\
\text { Copolymer }\end{array}$ & 625 & 625 & 625 & 625 & 625 \\
\hline $\begin{array}{l}\text { PANI+PS } \\
\text { blend }\end{array}$ & 625 & 625 & 625 & 625 & 312.5 \\
\hline
\end{tabular}

